\title{
Lomustine-temozolomide combination efficacious in newly diagnosed glioblastoma
}

The prognosis of patients with glioblastoma remains dismal, with median overall survival (OS) durations of $\sim 15$ months. New findings of a phase III trial indicate that adding lomustine to the standard treatment regimen improves the outcome of certain patients.

On the basis of evidence from a single-arm phase II study in 31 patients indicating an exclusive OS benefit, this phase III trial enrolled only patients with newly diagnosed MGMT promoter-methylated glioblastoma. In general, these patients have better responses to therapy and, thus, OS than those with $M G M T$ promoter-unmethylated disease. After surgery, patients received standard daily radiotherapy either with daily temozolomide followed by six cycles of adjuvant temozolomide $(n=63)$, or with one course of lomustine and temozolomide chemotherapy (during the first week) followed by five additional cycles of lomustine and temozolomide $(n=66)$.

Median OS was 48.1 months with the lomustine-temozolomide regimen versus 31.4 months with temozolomide alone
(HR 0.60, 95\% CI 0.35-1.03; $P=0.0492$ ).

In both groups, the median progression-free survival was 16.7 months $(P=0.4113)$.

The frequency of grade 3-4 adverse events occurring up to 30 days after the end of study treatment was higher with lomustinetemozolomide than with temozolomide alone (59\% versus $51 \%$ ). No treatment-related deaths were reported. Notably, lower proportions of patients in the experimental arm received all six courses of chemotherapy (39\% versus $60 \%$ ) and the maximum temozolomide dose (38\% versus $67 \%$ ).

The investigators acknowledge that further studies in large cohorts are needed to confirm their findings. However, the current data indicate that the lomustinetemozolomide regimen has the potential to become a new standard of care.

David Killock

ORIGINAL ARTICLE Herrlinger, U. et al. Lomustinetemozolomide combination therapy versus standard temozolomide therapy in patients with newly diagnosed glioblastoma with methylated MGMT promoter (CeTeG) NOA-09): a randomised, open-label, phase 3 trial. Lancet 393. 678-688 (2019)

\section{ARAMIS - is darolutamide set to become the 'third musketeer' of nmCRPC?}

The androgen receptor (AR) inhibitors apalutamide and enzalutamide prolong metastasis-free survival (MFS) and are currently approved for use in men with nonmetastatic castration-resistant prostate cancer (nmCRPC). Now, the results of the phase III ARAMIS trial of darolutamide suggest that this novel AR inhibitor is set to become an alternative option for these men.

ARAMIS involved 1,509 patients with a prostate-specific antigen (PSA) doubling time of $\leq 10$ months. The median MFS of the 955 men assigned to receive darolutamide plus continued androgen-deprivation therapy (ADT) was 40.4 months versus 18.4 months with placebo plus ADT (HR 0.41, 95\% Cl 0.34-0.50; $P<0.001$ ). In interim analyses of secondary or exploratory end points, darolutamide also improved the times to PSA progression, pain progression, chemotherapy or anti-neoplastic therapy and a symptomatic skeletal event, as well as progression-free and overall survival. In general, the ARAMIS data are consistent with those of the SPARTAN and PROSPER trials of apalutamide and enzalutamide, respectively, in similar patient cohorts.

\section{In the news}

\section{FROM TAT 2019}

The International Congress on Targeted Anticancer Therapies (TAT), dubbed 'the home of phase I oncology', was held for the seventeenth time this year and for the second year under the auspices of ESMO. The translational research and early clinical data presented at this engaging meeting are often a bellwether and driver of future advances in cancer therapy. This year, a number of key themes emerged.

In his opening address, the current ESMO President, Josep Tabernero, emphasized the ESMO 2020 Vision, with three main pillars: integrated cancer care, specialized education and sustainable cancer care. These aims were evident at TAT 2019 and resonate with the values of our journal.

Geoffrey Shapiro received the TAT 2019 Honorary Award and delivered a keynote lecture focused on the continuing clinical development of cyclin-dependent kinase (CDK) inhibitors, in which his work has been instrumental. He alluded to the potential of biomarker research to improve outcomes with CDK4/6 inhibitors, in the approved indications in breast cancer and beyond, as well as inhibitors of other CDKs. In addition, the need to consider the effects of these agents on the immune tumour microenvironment was underscored. Correspondingly, data presented by Gordon Freeman and Benjamin Izar demonstrated interactions between CDK4/6 activity and suppression of $T$ cell responses, thus highlighting the potential of CDK4/ 6 inhibitors as rational drug partners for immune-checkpoint inhibitors (ICls).

Indeed, the combination of $\mathrm{ICl}$ s with a range of other therapies was a recurring theme. However, an update from the Methodology for the Development of Innovative Cancer Therapies (MDICT) Task Force suggested that the sheer number of immunotherapies and related trials, particularly of combination therapies, is putting pressure on patient resources. Accordingly, the importance of rational combinations, novel preclinical models, and appropriate and maximally informative trial designs (including the inclusion of more adolescents and young adults) was discussed. Other notable topics included liquid biopsy approaches to enhancing the pharmacological audit trail, as well as novel strategies for targeting DNA repair, RAS-mutant cancers and oncogenic fusions (all of which have been covered extensively in this journal). We look forward to seeing these advances permeate into future clinical practice. 